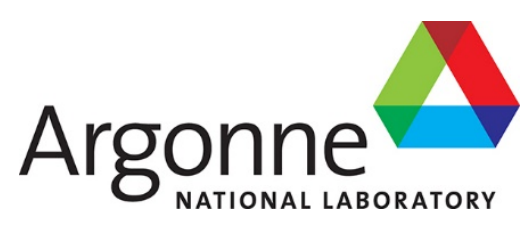

\title{
Preliminary Study on TRISO Fuel Cross Section Generation
}

Nuclear Science and Engineering Division 


\begin{abstract}
About Argonne National Laboratory
Argonne is a U.S. Department of Energy laboratory managed by UChicago Argonne, LLC under contract DE-AC02-06CH11357. The Laboratory's main facility is outside Chicago, at 9700 South Cass Avenue, Argonne, Illinois 60439. For information about Argonne and its pioneering science and technology programs, see www.anl.gov.
\end{abstract}

\title{
DOCUMENT AVAILABILITY
}

Online Access: U.S. Department of Energy (DOE) reports produced after 1991 and a growing number of pre-1991 documents are available free at OSTI.GOV (http://www.osti.gov/), a service of the US Dept. of Energy's Office of Scientific and Technical Information.

Reports not in digital format may be purchased by the public from the National Technical Information Service (NTIS):

U.S. Department of Commerce

National Technical Information Service

5301 Shawnee Rd

Alexandra, VA 22312

www.ntis.gov

Phone: (800) 553-NTIS (6847) or (703) 605-6000

Fax: (703) 605-6900

Email: orders@ntis.gov

Reports not in digital format are available to DOE and DOE contractors from the Office of Scientific and Technical Information (OSTI):

U.S. Department of Energy

Office of Scientific and Technical Information

P.O. Box 62

Oak Ridge, TN 37831-0062

www.osti.gov

Phone: (865) 576-8401

Fax: (865) 576-5728

\footnotetext{
Disclaimer

This report was prepared as an account of work sponsored by an agency of the United States Government. Neither the United States Government nor any agency thereof, nor UChicago Argonne, LLC, nor any of their employees or officers, makes any warranty, express or implied, or assumes any legal liability or responsibility for the accuracy, completeness, or usefulness of any information, apparatus, product, or process disclosed, or represents that its use would not infringe privately owned rights. Reference herein to any specific commercial product, process, or service by trade name, trademark, manufacturer, or otherwise, does not necessarily constitute or imply its endorsement, recommendation, or favoring by the United States Government or any agency thereof. The views and opinions of document authors expressed herein do not necessarily state or reflect those of the United States Government or any agency thereof, Argonne National Laboratory, or UChicago Argonne, LLC.
} 


\section{Preliminary Study on TRISO Fuel Cross Section Generation}

prepared by

Hansol Park, Won Sik Yang, Changho Lee, Yeon Sang Jung

Nuclear Science and Engineering Division, Argonne National Laboratory

September 30, 2020 



\begin{abstract}
Cross section self-shielding methodologies for TRISO fuel were assessed to provide accurate multigroup cross sections for a high-fidelity reactor physics code so that the code is able to accurately model and simulate advanced reactors with TRISO fuel. Initially, the two existing methodologies (the SCALE method and the Sanchez-Pomraning method) were studied and implemented to $\mathrm{MC}^{2}-3$ for detailed performance tests. Additionally, a new spatial self-shielding method, named the iterative local spatial self-shielding (ILSS) method, for particulate fuels was developed based on the disadvantage factor and implemented to $\mathrm{MC}^{2}-3$ as well. The new method approximately accounts for the effect of randomly distributed particles on the particle shadowing effect using a homogenized compact region surrounding a particle of interest at the center. The self-shielded cross sections of the particle at the center are determined iteratively since the cross sections of the homogenized compact region are calculated using them. For the energy range above $100 \mathrm{keV}$ where the fuel-to-moderator ratio is more important than the random distribution of particles, a single particle unit-cell model is used by preserving the average amount of moderator per fuel particle in the system.
\end{abstract}

The three self-shielding methods implemented in $\mathrm{MC}^{2}-3$ were tested using numerical benchmark problems made based on fuel compact problems of a prismatic-type very high temperature reactor. Test results indicated that the ILSS method produced slightly better results than the SCALE and Sanchez-Pomraning methods, compared to the Serpent-2 Monte Carlo results obtained with 25 independent random particle configurations. The SCALE and SanchezPomraning methods tend to underestimate the heterogeneity effect by 150 and $100 \mathrm{pcm}$, respectively, while the new ILSS method overestimates the heterogeneity effect by $70 \mathrm{pcm}$.

In future, the new self-shielding method will be extended to perform pebble calculations and compare results with those from the SCALE and Sanchez-Pomraning methods. Furthermore, the new method will be optimized for practical applications to on-the-fly resonance treatment for lattice or whole-core calculations for advanced reactors. 


\section{TABLE OF CONTENTS}

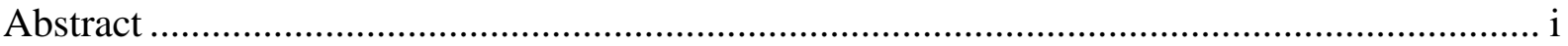

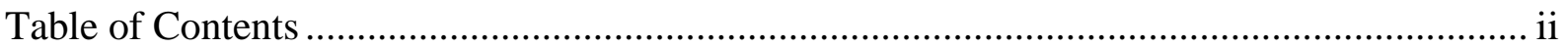

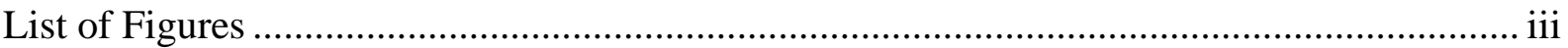

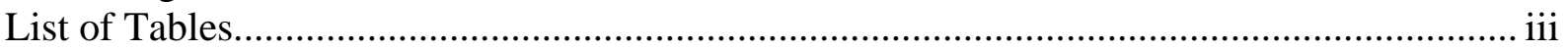

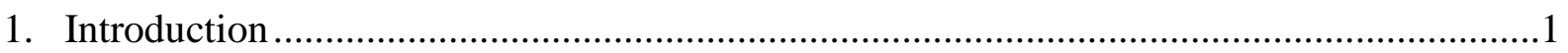

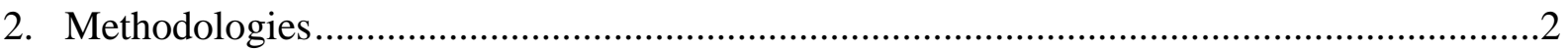

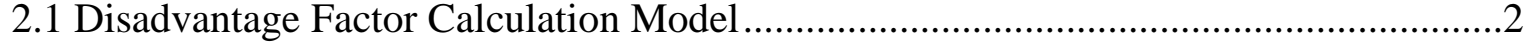



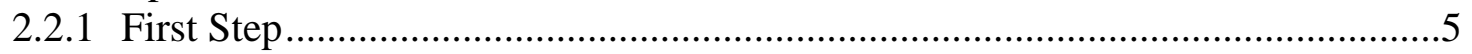

2.2.2 Second Step ....................................................................................

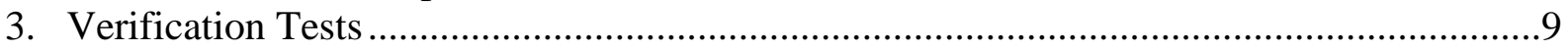

3.1 Problem Specifications and Serpent-2 Solutions .................................................

3.2 Sensitivity Study to Determine Optimum Parameters for $\mathrm{MC}^{2}-3$ MOC Calculation ...10

3.3 Comparison of Eigenvalue Results ............................................................... 12

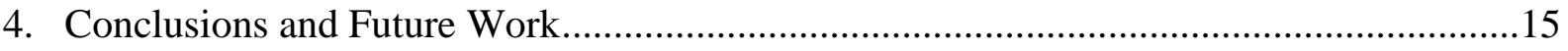

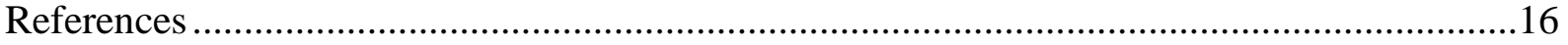




\section{LIST OF FIGURES}

Figure 2-1. A Single Particle Unit-Cell Model for Calculating DFs for Energy Range above $100 \mathrm{keV}$.

Figure 2-2. A Single Particle Unit-Cell Model for Calculating DFs for Energy Range below $100 \mathrm{keV}$. 4

Figure 2-3. Pseudocode for Initial UFG Eigenvalue Calculation. .......................................... 5

Figure 2-4. Pseudocode for HFG Fixed Source Calculation with DF Iteration........................ 6

Figure 2-5. Pseudocode for UFG Eigenvalue Calculation with DF Iteration......................... 7

Figure 3-1. Fuel Particle and Compact Design Parameters of NGNP Design [10].................. 9 Figure 3-2. Cell-Averaged 62-Group Neutron Spectrum in Heterogeneous Unit Compact-Cell

vs. Fuel Particle Packing Fraction............................................................................. 11

Figure 3-3. Super-Cell Problem of 2 Rings of Identical Hexagonal Compact-Cells............... 11

Figure 3-4. Comparison of Eigenvalues of $\mathrm{MC}^{2}-3$ HFG Calculation with Transport Corrected $\mathrm{P}_{0}$ Scattering with Serpent-2 Eigenvalue. .............................................................. 13

\section{LIST OF TABLES}

Table 3-1. Packing Fraction and Number of Fuel Particles .................................................. 9

Table 3-2. Serpent-2 Eigenvalue Results of 2D Unit Fuel Compact-Cell Problems ............... 10

Table 3-3. MC $^{2}-3$ Eigenvalue Results vs. Radial Meshes of Unit-Cell Problem ................... 12

Table 3-4. MC M $^{2} 3$ Eigenvalue Results of Super-Cell Problem .............................................. 12

Table 3-5. Results of $\mathrm{MC}^{2}-3$ UFG Calculations with Transport Corrected $\mathrm{P}_{0}$ Option for Fuel Compact Super-Cell Problems

Table 3-6. Results of MC $\mathrm{MC}^{2}-3$ HFG Calculations with Transport Corrected $\mathrm{P}_{0}$ Option for Fuel Compact Super-Cell Problems 



\section{Introduction}

Under the DOE-NE NEAMS program, a high-fidelity reactor physics code has been developed to support the users for various-type advanced reactors. The development of the high-fidelity reactor physics codes has focused largely on neutron transport calculation, multigroup cross section generation, and depletion calculation capabilities. In recent years, with increased interests in the use of TRISO (TRi-structural ISOtropic) particle fuels for advanced reactors including micro reactors, the need for accurate cross section generation is increasing accordingly. The most important element to meet the need is to supply accurate cross sections to the reactor physics codes. The cross section API (CSAPI) [1] has been developed and plugged into PROTEUS and recently Griffin, however a capability of treating such particulate fuel types is missing. Therefore, it was decided to assess the existing methodologies of generating TRISO fuel cross sections, develop a new methodology if necessary, implement and test them in $\mathrm{MC}^{2}-3$, and provide a path forward to improve the CSAPI with the cross section methodology for the high-fidelity reactor physics code so that the code is able to model and simulate advanced reactors with TRISO particulate fuels.

Conventional High Temperature Gas-Cooled Reactors (HTGRs) and many of recent micro-reactor concepts use TRISO fuel grains embedded in a ceramic or metal matrix. These particulate fuels are characterized by the double heterogeneity caused by randomly dispersed fuel particles in matrix in cylindrical compact or pebble geometry which are heterogeneously arranged in the core. The double heterogeneity effect should be accounted for in generating multigroup cross sections with proper energy and spatial self-shielding of resonances to obtain accurate neuron transport solutions. A simple volume-averaging approach would lead to significant underestimation of the multiplication factor of thermal reactors due to the neglect of spatial self-shielding in fuel particles. Thus, a proper method to treat the double heterogeneity effect should be implemented in lattice codes for multigroup cross section generation. Motivated by these needs, work was initiated to implement the methods which are able to treat the double heterogeneities of particulate fuels in $\mathrm{MC}^{2}-3$ [2].

The main objective of the double heterogeneity treatment in $\mathrm{MC}^{2}-3$ is to provide homogenized fuel compact cross sections to subsequent down-stream calculations such as the assembly lattice calculations in $\mathrm{MC}^{2}-3$ or the heterogeneous whole-core calculations with PROTEUS [3] or Griffin [4]. In this work, three approaches were implemented for comparison: 1) the existing SCALE [5] method, 2) the Sanchez-Pomraning [6,7] method, and 3) a new iterative local spatial self-shielding (ILSS) approach involving homogenization. The SCALE and ILSS methods employ a two-step procedure in which compact-homogenized ultrafine group (UFG) and hyperfine group (HFG) microscopic cross sections are computed at the first step and a fuel compact lattice calculation is performed using these UFG and HFG cross sections at the second step. The Sanchez-Pomraning method directly performs the fuel compact lattice calculation with the cross sections of each layer of fuel particle and those of matrix in a fuel compact using the method of characteristics (MOC) modified by the Sanchez-Pomraning approach.

The purpose of this report is to present the preliminary performance of $\mathrm{MC}^{2}-3$ for two-dimensional (2D) unit- and super-cell problems of particulate fuel compacts for a prismatic-type very high temperature reactor (VHTR). Section 2 presents the new ILSS method. In Section 3, verification test results for the three self-shielding methods are provided using 2D benchmark problems. Conclusions and future work are discussed in Section 4. 


\section{Methodologies}

One of the representative existing methods to treat double heterogeneity is the SCALE method [5] in which the two-step calculation is employed. In the first step, the disadvantage factors (DFs) of fuel particles are calculated by solving a fixed source point-wise slowing down problem with the CENTRM code [8] for a single particle unit-cell that preserves a packing fraction. The DF is defined for each mesh in the unit-cell problem as the ratio of the mesh flux to the cell-averaged flux. Then, using the DFs, cell-averaged cross sections are calculated as

$$
\tilde{\sigma}_{X, g}^{i}=\sum_{k} v_{k} N_{k}^{i} d_{g, k} \sigma_{X, g, k}^{i} / \tilde{N}^{i}
$$

where $X, g, i$ and $k$ are the indices of reaction type, energy group, isotope and mesh of the problem, respectively, tilde means the average value for the problem domain, $v_{k}$ is the volume fraction of mesh $k$, and $d_{g, k}$ is the DF of mesh $k$. These average cross sections are taken as average quantities of a compact. These compact-averaged point-wise cross sections are used to solve a lattice problem in the second CENTRM calculation and the resulting compact-averaged point-wise spectrum is used to condense the point-wise cross sections into a broad group structure.

When calculating the DFs to obtain compact-averaged cross sections, the SCALE method assumes the regular array of particles which leads to more neutron collisions in fuel particles, resulting in a slight underestimation of the reactivity. In order to improve the model by accounting for the effect of randomly distributed particles on the particle shadowing effect, a new method was proposed in this study.

The newly developed scheme named the iterative local spatial self-shielding (ILSS) method employs the same two-step procedure as in the SCALE approach. The difference of the ILSS method from the SCALE approach is the different model used to calculate DFs in the first step. While the SCALE method solves the unit-cell problem of a single particle surrounded by the matrix material to calculate DFs, the ILSS method uses two different unit-cell models depending on the neutron energy. In the fast energy range where the mean free path is much larger than the average distance between particles, the fuel-to-moderator ratio affects the DF more than the random distribution of particles. Thus, for the energy range above $100 \mathrm{keV}$, the unit-cell problem is made of a single fuel particle surrounded by the matrix material and the moderator outside of the fuel compact. For the energy range below $100 \mathrm{keV}$ where the mean free path is shorter than or comparable to the average distance between particles, the unit-cell is made of a single fuel particle surrounded by a homogenized compact. Since the homogenized compact contains the same fuel particles as the central particle, the DFs of the central particle are used in determining the homogenized compact cross sections and thus the DFs are calculated iteratively. This section describes the DF calculation models as well as the overall procedure of the ILSS method.

\subsection{Disadvantage Factor Calculation Model}

Two different single particle unit-cell models are used to calculate DFs depending on neutron mean free path (neutron energy). For the energy range above $100 \mathrm{keV}$, a single particle unit-cell model shown in Figure 2-1 is used. A target particle with its explicit internal geometry is located at the center and surrounded by the matrix material and the moderator outside of the fuel compact. If there are $N(>1)$ types of particles, the matrix region in Figure 2-1 becomes the homogenized mixture of the matrix and other types of particles. Thus, $N$ independent single particle unit-cell problems are 
solved and DFs in energy group $g$ for particle type $n$ defined in Eq. (2) for each particle type are obtained.

$$
d_{g, n}=\frac{\phi_{g, n}^{[n]}}{\tilde{\phi}_{g}^{[n]}},
$$

where $\phi_{g, n}^{[n]}$ and $\tilde{\phi}_{g}^{[n]}$ are the particle flux and the flux averaged over the particle and the matrix for the particle type $n$ problem, respectively. Using these particle DFs at the end of $N$ independent single particle problem calculations, the matrix DFs are obtained by

$$
d_{g, 0}=\left(1-\sum_{n=1}^{N} v_{n} d_{g, n}\right) / v_{0},
$$

where $v_{0}$ and $v_{n}$ are the volume fractions of the matrix and particle type $n$, respectively. For each particle type problem, the size of the matrix is determined to preserve the volume packing fraction of the center particle type in the fuel compact. The material of the outer moderator region is the homogenized composition of the outside graphite moderator and coolant such as helium or molten salt. The size of the outer moderator region is determined to preserve the relative amount to the center particle. Note that the SCALE approach uses a similar model without the outer moderator region.



Figure 2-1. A Single Particle Unit-Cell Model for Calculating DFs for Energy Range above $100 \mathrm{keV}$.

For the energy range below $100 \mathrm{keV}$, a different single particle unit-cell model is used as shown in Figure 2-2. A target particle is located at the center with its explicit internal geometry and the effect of randomly distributed particles on the particle-to-particle Dancoff factor is considered by representing a random configuration of particles as a homogenized compact. Note that there is no outer moderator because the spatial self-shielding in the center particle cannot be correctly considered via the outer moderator located far from the center particle. Since the cross sections of the homogenized compact region is determined by the self-shielded cross sections of the center 
particle, the problem should be solved iteratively until the self-shielding condition of the center particle converges. The cross sections of the homogenized compact are calculated as

$$
\sum_{X, g, H . C .}^{[n]}=\sum_{n^{\prime}=0}^{N} \sum_{i \in n^{\prime}} \tilde{N}_{n^{\prime}}^{i[n]} \sigma_{X, g, n^{\prime}}^{i} d_{g, n^{\prime}}
$$

where H.C. denotes the homogenized compact, $N$ is the number of different particle types, the particle type 0 denotes the matrix, and $\tilde{N}_{n^{\prime}}^{i[n]}$ is the isotope $i$ number density of particle type $n^{\prime}$ in the homogenized compact region for a particle type $n$ problem. It should be noted that the DFs of the matrix should be updated as in Eq. (3) when each particle type problem is solved. As $d_{g, n}$ depends on the macroscopic cross section of the homogenized compact region in Eq. (4), the coupled set of $N$ particle problems should be solved iteratively until all $d_{g, n}$ for $n \leq N$ converge. Note that even the case of $N=1$ needs iteration due to the nonlinearity in Eqs. (2) to (4). In contrast, the cross sections of the homogenized mixture for $N>1$ in the single particle unit-cell model for the energy range above $100 \mathrm{keV}$ are computed without the DF in Eq. (4), and thus $N$ problems are independent of each other and accordingly no iterations are needed.

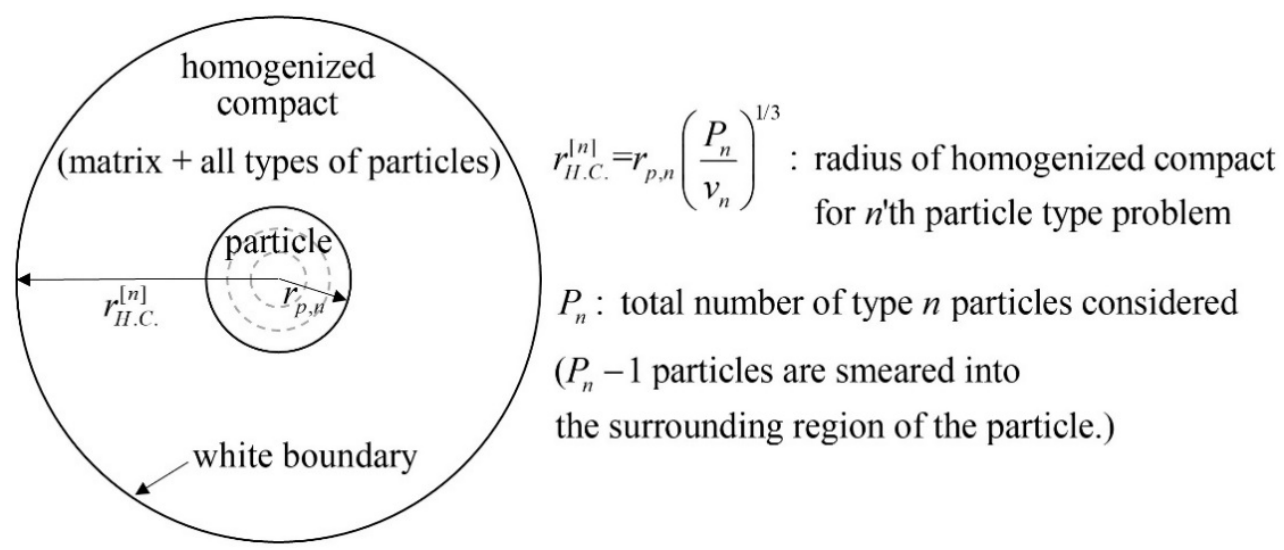

Figure 2-2. A Single Particle Unit-Cell Model for Calculating DFs for Energy Range below $100 \mathrm{keV}$.

Isotope number densities in the homogenized compact region are determined to preserve the total number of isotopes in a compact as

$$
\begin{aligned}
& \tilde{N}_{n^{\prime}}^{i[n]}=N_{n^{\prime}}^{i[n]} v_{n^{\prime}} \frac{1}{1-v_{n} / P_{n}} \text { for } n^{\prime} \neq n \text {, including } 0 \text { for matrix, } \\
& \tilde{N}_{n}^{i[n]}=N_{n}^{i[n]} v_{n} \frac{1-1 / P_{n}}{1-v_{n} / P_{n}}
\end{aligned}
$$

where $P_{n}$ is the total number of type $n$ particles in a compact. Since one particle is located at the center, $P_{n}-1$ particles are smeared into the homogenized compact region to model randomly dispersed neighboring particles. Note that in the single particle unit-cell model for the energy range above $100 \mathrm{keV}, P_{n}=1$, and thus no target particles are present in the outside region of the center particle. Numerical tests showed that the calculated DFs converges as the size of homogenized 
region (i.e., $P_{n}$ for given packing fraction) increases and a practical convergence can be obtained with $P_{n}=100$.

\subsection{Two-step Calculation Procedure}

The overall procedure is divided into two steps. In the first step, for all particles and the matrix, HFG DFs are calculated for resolved resonance energy ranges and UFG DFs are calculated for the whole energy range. Using these DFs, compact-averaged UFG and HFG cross sections are computed. Then, in the second step, the normal $\mathrm{MC}^{2}-3$ UFG/HFG calculations are performed to account for the second heterogeneity using the compact-averaged cross sections.

\subsubsection{First Step}

In the first step, DFs are obtained through three step calculations. First, UFG DFs for the energy range above $100 \mathrm{keV}$ are obtained by performing $N$ independent UFG eigenvalue calculations using the single particle unit-cell model in Figure 2-1. The other purpose of this step is to obtain compactaveraged flux solutions to calculate fixed fission and inelastic scattering sources in the subsequent HFG fixed source calculation. Figure 2-3 shows the pseudocode for the initial UFG eigenvalue calculation. This step calculation follows the normal MC²-3 UFG eigenvalue calculation procedure with the additional routine to calculate DFs.

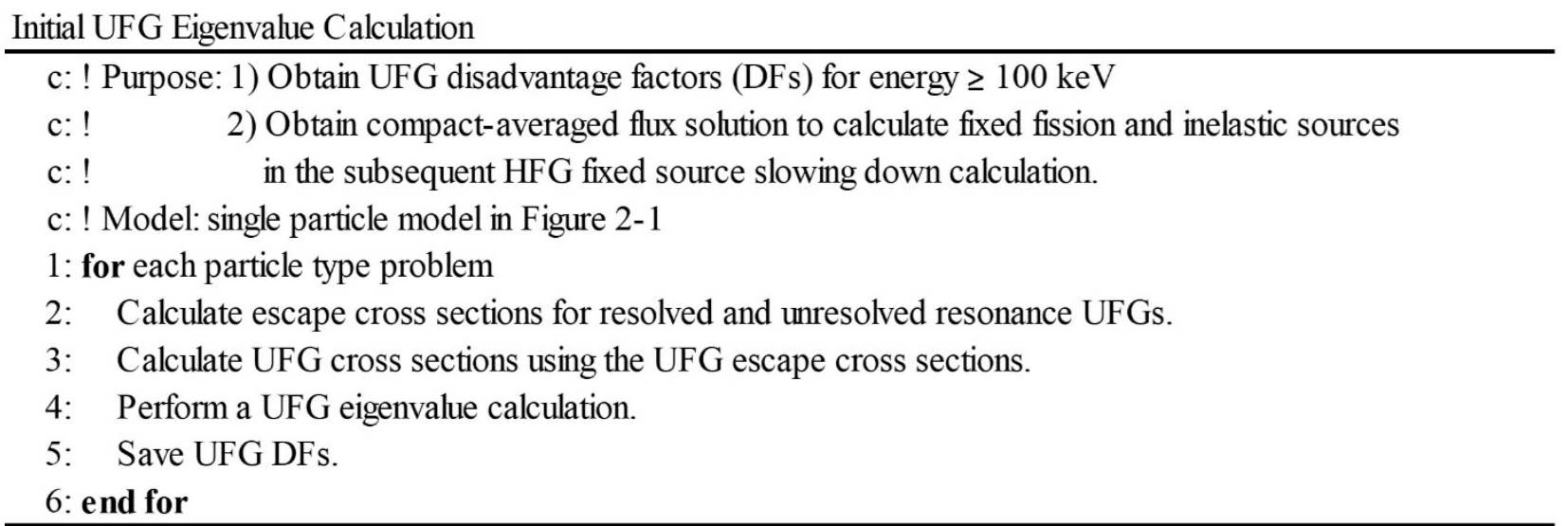

Figure 2-3. Pseudocode for Initial UFG Eigenvalue Calculation.

Second, as shown in Figure 2-4, HFG DFs for resolved resonance energy ranges are obtained by performing the HFG fixed source calculation with the DF iteration at each HFG. At each HFG, the fission and inelastic scattering sources in the center particle and the homogenized compact are calculated by interpolating the UFG sources for fuel particles

$$
\begin{gathered}
Q_{f, g, n}=\sum_{g^{\prime}} \phi_{g^{\prime}, n}^{[n]} \sum_{i \in n} N_{n}^{i} \chi_{g^{\prime} \rightarrow g}^{i} v \sigma_{f, g^{\prime}, n^{\prime}}^{i}, \\
Q_{\text {inel, }, g, n}=\sum_{g^{\prime}} \phi_{g^{\prime}, n}^{[n]} \sum_{i \in n} N_{n}^{i} \sigma_{\text {inel }, g^{\prime} \rightarrow g, n^{\prime}}^{i},
\end{gathered}
$$

and those for the homogenized compact 


$$
\begin{aligned}
& Q_{f, g, H . C .}=\sum_{n^{\prime}=0}^{N} \sum_{g^{\prime}} d_{g^{\prime}, n^{\prime}} \tilde{\phi}_{g^{\prime}}^{[n]} \sum_{i \in n^{\prime}} \tilde{N}_{n^{\prime}}^{i[n]} \chi_{g^{\prime} \rightarrow g}^{i} v \sigma_{f, g^{\prime}, n^{\prime}}^{i}, \\
& Q_{\text {inel,g,H.C. }}=\sum_{n^{\prime}=0}^{N} \sum_{g^{\prime}} d_{g^{\prime}, n^{\prime}} \tilde{\phi}_{g^{\prime}}^{[n]} \sum_{i \in n^{\prime}} \tilde{N}_{n^{\prime}}^{i[n]} \sigma_{i n e l, g^{\prime} \rightarrow g, n^{\prime} \cdot}^{i}
\end{aligned}
$$

Unlike in the initial UFG DF calculation that calculates DFs after the UFG eigenvalue calculation for each particle type problem, the HFG slowing down calculation is performed only once with the DF iteration loop inside the energy point (HFG) loop. That is, the DF iteration is done by solving $N$ coupled particle problems via Eqs. (2) to (4) using a given down-scattering source at each HFG. These HFG DFs will be used to compute compact-averaged HFG cross sections. Once HFG flux solutions at each micro-region of particles and the matrix are obtained, UFG cross sections at the corresponding regions are updated with the HFG spectrum solution.

HFG Fixed Source DF Iteration

c: ! Purpose: 1) Obtain HFG DFs at resolved resonance HFGs.

c: ! $\quad 2)$ Obtain self-shielded UFG cross sections at each microregion of particles and a matrix.

c: ! Model: single particle model in Figure 2-2

1: for each particle type problem

2: Determine number densities of isotopes at the homogenized compact region. (Eq. (5))

3: Obtain HFG macroscopic cross sections at the homogenized compact region without HFG DFs.

4: Calculate fixed fission and inelastic scattering sources at all HFGs using UFG flux solutions and

UFG DFs obtained from the initial UFG eigenvalue calculation. (Eqs. (6) and (7))

\section{5: end for}

6: for each HFG

7: for DF iteration

8: $\quad$ for each particle type problem

9: Update macroscopic cross sections at the homogenized compact region using the most recent DFs. (Eq. (4))

10: Calculate collision probabilities with the white boundary condition.

11: Calculate HFG source. (only at the first DF iteration)

12: Obtain HFG flux solution and update HFG DFs of the current particle and the matrix.

13: $\quad$ end for

14: end for

15: end for

Figure 2-4. Pseudocode for HFG Fixed Source Calculation with DF Iteration.

With these UFG cross sections, the UFG eigenvalue calculation modified with DF iterations is performed at the third step as shown in Figure 2-5. The fission sources used in the HFG fixed source calculation step are taken as the initial fission source distributions. Then, the fission source iteration is performed with modifications of the normal routine. For a given fission source distribution, the entire group sweep for each of $N$ particle problems is performed sequentially with the sequential update of UFG DFs of the current particle type and the matrix for the energy range below $100 \mathrm{keV}$. That is, after the group sweep with a given fission source distribution, DFs are updated, and thus 
the macroscopic cross sections of the homogenized composition region are updated. Then, the fission source and eigenvalue are updated using the updated macroscopic cross sections of the homogenized composition region for the next fission source iteration step. The fission source iteration continues until the relative eigenvalue errors and the eigenvalue differences among all particle type problems converge. Using the converged UFG DFs, compact-averaged interim UFG cross sections are calculated using Eq. (1). Note that these UFG cross sections are not true compactaveraged quantities since the compact escape probability has not been considered yet.

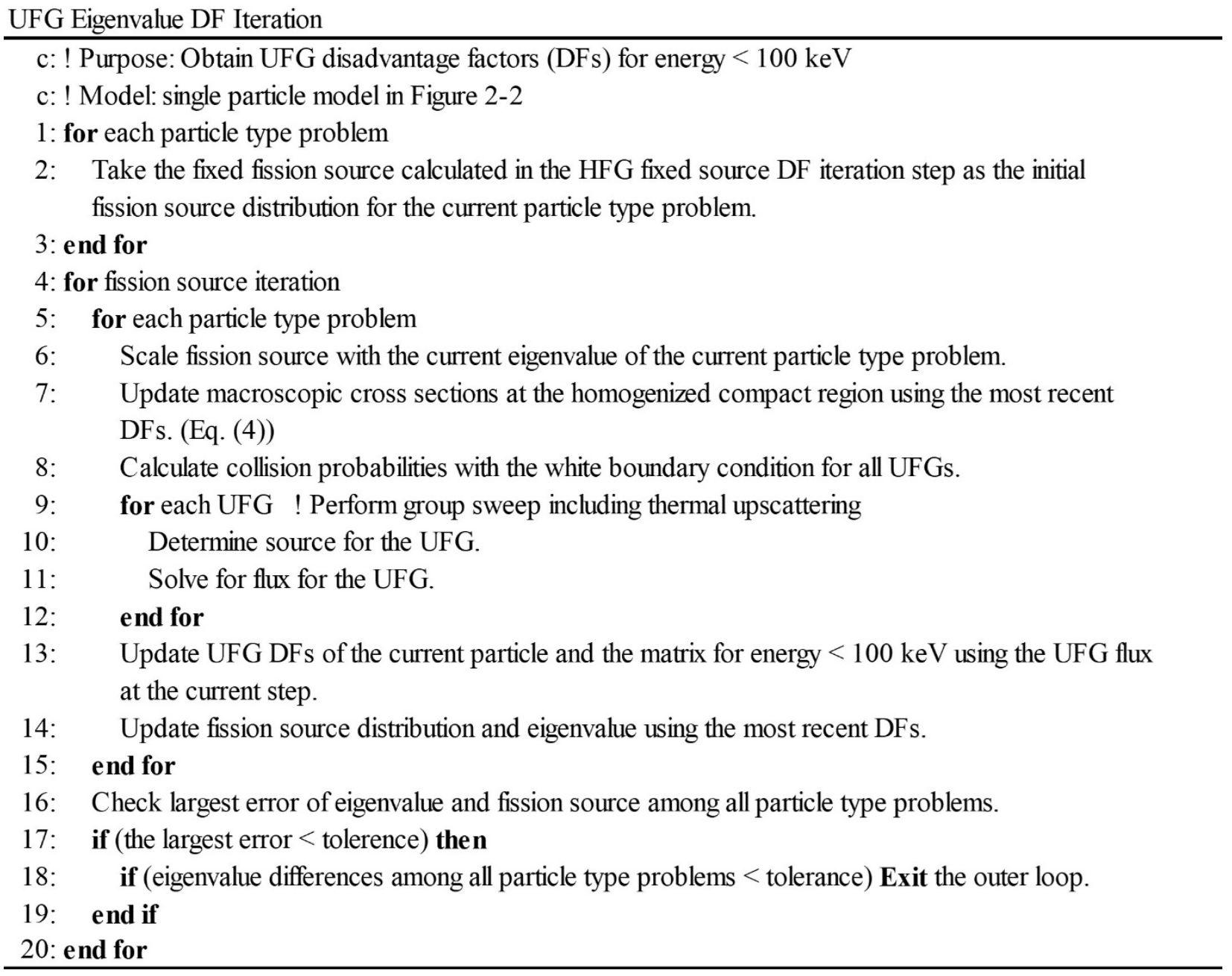

Figure 2-5. Pseudocode for UFG Eigenvalue Calculation with DF Iteration.

\subsubsection{Second Step}

The second step calculation procedure is the same as the normal $\mathrm{MC}^{2}-3$ calculation procedure. Using compact-averaged interim UFG total cross sections calculated the first step, the escape cross sections for each UFG are obtained as 


$$
\tilde{\Sigma}_{e, g, k}^{i}=\frac{\sum_{j} \tilde{P}_{g, j \rightarrow k} V_{j} \sum_{m \neq i} \tilde{\Sigma}_{t, g, j}^{m}}{\sum_{j} \tilde{P}_{g, j \rightarrow k} V_{j} \tilde{N}_{j}^{i} / \tilde{N}_{k}^{i}}-\sum_{m \neq i} \tilde{\Sigma}_{t, g, k}^{m},
$$

where $k$ and $j$ are region indices, $i$ and $m$ are isotope indices, $g$ refers to an UFG, and the tilde sign means a compact-averaged quantity. Note that the collision probabilities are calculated using the compact-averaged UFG total cross sections. Since UFG total cross sections depend on the escape cross sections, the escape cross sections should be obtained iteratively. Tests indicated that less than six iterations were sufficient for convergence in most cases. With the obtained escape cross sections, the HFG flux spectrum in Eq. (9) based on the NR approximation is used as a weighting function to calculate the compact-averaged UFG cross sections that consider the compact escape probability at resolved resonance energy range.

$$
\phi_{h f g, k}^{i}=\Delta u_{h f g} \frac{C}{\sum_{m} \tilde{N}_{k}^{m} \tilde{\sigma}_{t, h f g, k}^{m}+\tilde{\Sigma}_{e, g, k}^{i}},
$$

where $\Delta u_{h f g}$ is the lethargy width of a HFG (=1/24000). For the unresolved resonance energy range, the escape cross section in Eq. (8) is used in the formula of the unresolved resonance cross section in $\mathrm{MC}^{2}-3$.

Numerical tests indicated that the use of approximated UFG cross sections induced at least 100 pcm errors for middle and high packing fraction problems. Thus, using the fission and inelastic scattering sources interpolated from the UFG eigenvalue calculation results, the HFG fixed source calculation is performed with the compact-averaged HFG cross sections calculated at the first step to obtain more accurate HFG spectrum within a UFG. Using the compact-averaged HFG spectrum solution, more accurate compact-averaged UFG cross sections are obtained. Then, another UFG eigenvalue calculation is performed and the resulting UFG spectrum is used as a weighting function to condense compact-averaged UFG cross sections into a broad group structure. These compactaveraged broad group cross sections are then used in subsequent downstream calculations such as the assembly lattice calculations or heterogeneous whole-core calculations with PROTEUS or Griffin. 


\section{Verification Tests}

The newly developed ILSS method has been verified against the Serpent-2 code [9]. The first subsection describes the problem specification and the Serpent- 2 reference solutions. The second subsection shows the results of sensitivity test to determine optimum ray tracing parameters for $\mathrm{MC}^{2}-3 \mathrm{MOC}$ calculations, followed by comparison of results.

\subsection{Problem Specifications and Serpent-2 Solutions}

Eight hexagonal unit-cell problems were constructed by varying the fuel particle packing fraction. Figure 3-1 shows the cell configuration and the fuel particle and compact design parameters taken from the NGNP design [10]. Table 3-1 shows the fuel particle packing fractions and the number of particles. The number of particles in the table denotes that in a cylindrical compact of $0.6225 \mathrm{~cm}$ radius and $4.93 \mathrm{~cm}$ height, which was used only for Serpent-2 simulations. To evaluate the heterogeneity effect of fuel particles, a homogenized compact problem was also solved for each problem.



\begin{tabular}{ccccc}
\hline & Region & Radius $(\mathrm{cm})$ & Material & Density $\left(\mathrm{g} / \mathrm{cm}^{3}\right)$ \\
\hline & Fuel Kernel & 0.0175 & 10.36 wt.\% UCO & 10.5 \\
Fuel & Buffer & 0.0275 & Graphite & 1.00 \\
Particle & Inner PyC & 0.0310 & Graphite & 1.90 \\
& SiC & 0.0345 & SiC & 3.20 \\
& Outer PyC & 0.0385 & Graphite & 1.90 \\
\hline \multicolumn{2}{c}{ Fuel Compact } & 0.6225 & Graphite & 1.1995 \\
\hline \multicolumn{2}{c}{ Coolant } & 0.6350 & Helium & 0.0032 \\
\hline \multicolumn{2}{c}{ Graphite Block } & - & Graphite & 1.74 \\
\hline
\end{tabular}

Figure 3-1. Fuel Particle and Compact Design Parameters of NGNP Design [10].

Table 3-1. Packing Fraction and Number of Fuel Particles

\begin{tabular}{|c|c|c|}
\hline Problem Number & Packing Fraction (\%) & Number of Particles* \\
\hline 1 & 1.9914 & 500 \\
2 & 3.9829 & 1000 \\
3 & 9.9572 & 2500 \\
4 & 19.9143 & 5000 \\
5 & 27.8800 & 7000 \\
6 & 37.8372 & 9500 \\
7 & 47.7943 & 12000 \\
8 & 57.7515 & 14500 \\
\hline
\end{tabular}

* Number of particles in a cylindrical compact of $0.6225 \mathrm{~cm}$ radius and $4.93 \mathrm{~cm}$ height. 
Table 3-2 presents the Serpent-2 eigenvalue results. Homogenized compact problems were simulated using $10^{5}$ neutrons per cycle and 100 inactive and 1,000 active cycles. Each heterogeneous problem solution was determined by the statistical sample average of 25 independent simulation results with different sets of particle locations. The number of neutrons per cycle was $10^{5}$ and 100 inactive and 100 active cycles were used for solving the heterogeneous problems. The standard deviation of eigenvalue in each Monte Carlo simulation was between 2 to $7 \mathrm{pcm}$ and the sample standard deviation of 25 independent simulations for stochastic dispersion of fuel particles was between 5 to $13 \mathrm{pcm}$. The heterogeneity effect was calculated by the reactivity difference between the heterogeneous and homogenized compact problems. All the results in Table 3-2 were taken from the implicit estimator in the Serpent-2 outputs. The multiplication factor decreases with increasing packing fraction due to decreasing moderation as indicated by the spectrum change in Figure 3-2. The heterogeneity effect increases with increasing packing fraction, attaining a maximum at around a packing fraction of $15 \%$, and then decreases.

Table 3-2. Serpent-2 Eigenvalue Results of 2D Unit Fuel Compact-Cell Problems

\begin{tabular}{|c|cc|ccc|c|}
\hline \multirow{2}{*}{$\begin{array}{c}\text { Packing } \\
\text { Fraction (\%) }\end{array}$} & \multicolumn{2}{|c|}{ Homogeneous Compact } & \multicolumn{3}{|c|}{ Heterogeneous Compact } & \multirow{2}{*}{ Het. Effect } \\
\cline { 2 - 5 } K-eff $^{\text {pcm) }}$
\end{tabular}

a Standard deviation of one Monte Carlo simulation

b Sample standard deviation of 25 independent Monte Carlo simulations

\subsection{Sensitivity Study to Determine Optimum Parameters for $M^{2} C^{2}-3$ MOC Calculation}

The MOC is used to solve the transport equation for hexagonal unit-cell problems. To determine appropriate number of meshes and ray tracing parameters, a sensitivity study was performed with a fuel particle packing fraction of $47.808 \%$. The ray effect was also examined by solving the equivalent super-cell problems of 2 rings of identical hexagonal compact-cells shown in Figure 33 and comparing the results with those of the unit-cell problems.

Tables 3-3 and 3-4 show the $\mathrm{MC}^{2}-3$ eigenvalue results for unit- and super-cell problems, respectively, for different combinations of equal volume radial meshes in fuel compact and graphite moderator, azimuthal angles, and ray spacing. Different UFG cross sections were used for the radial meshes in fuel compact. The $\mathrm{P}_{1}$ scattering option was used and a convergence criterion of $10^{-6}$ was used for both eigenvalue and fission source. It was observed that the ray effect caused small reactivity differences between unit- and super-cell results. For example, the results for the case of 30 azimuthal angles in 180 degrees and ray spacing of $0.01 \mathrm{~cm}$ (with 5 radial meshes in each of fuel compact and moderator) showed a reactivity difference of $39 \mathrm{pcm}$. 


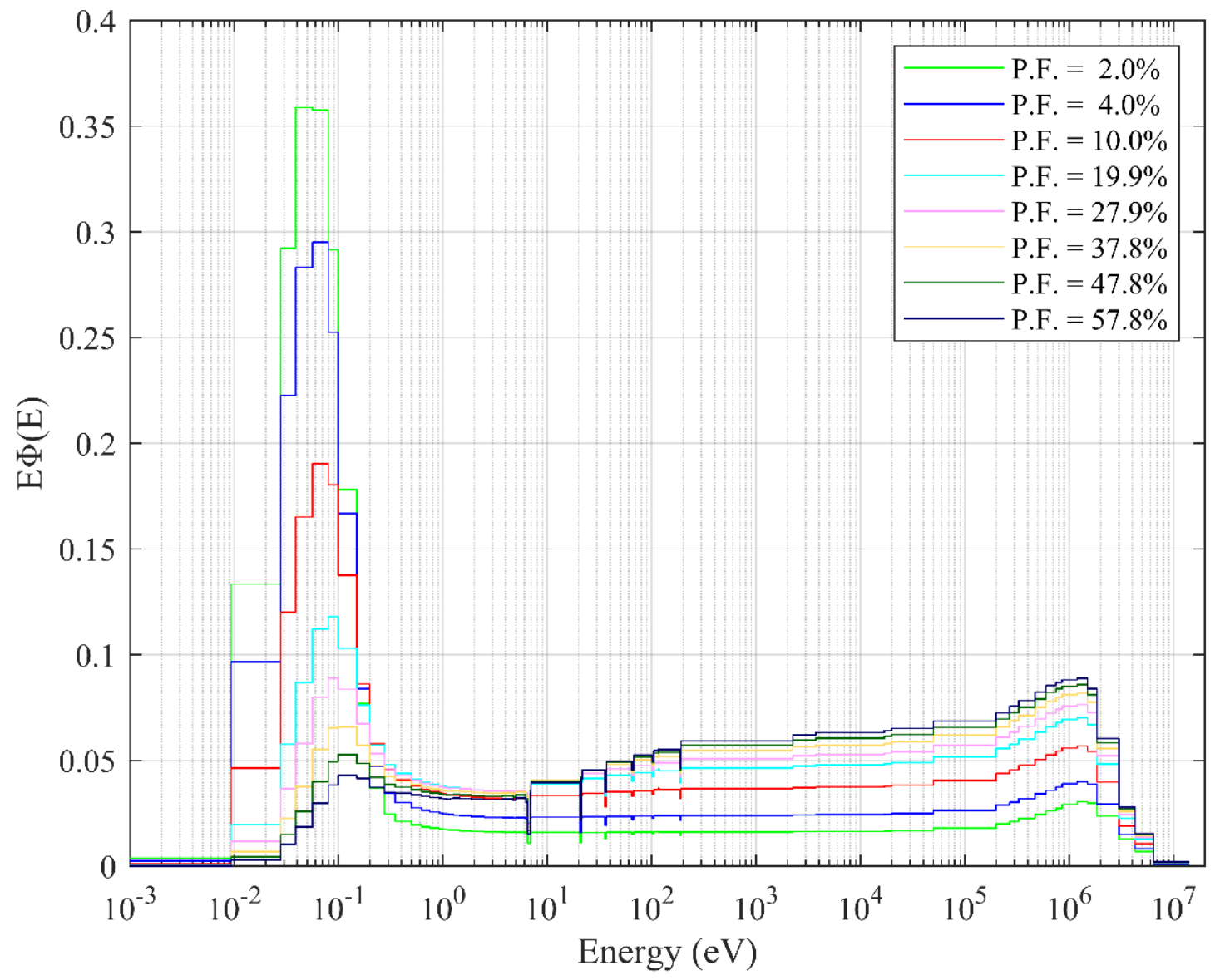

Figure 3-2. Cell-Averaged 62-Group Neutron Spectrum in Heterogeneous Unit Compact-Cell vs. Fuel Particle Packing Fraction.

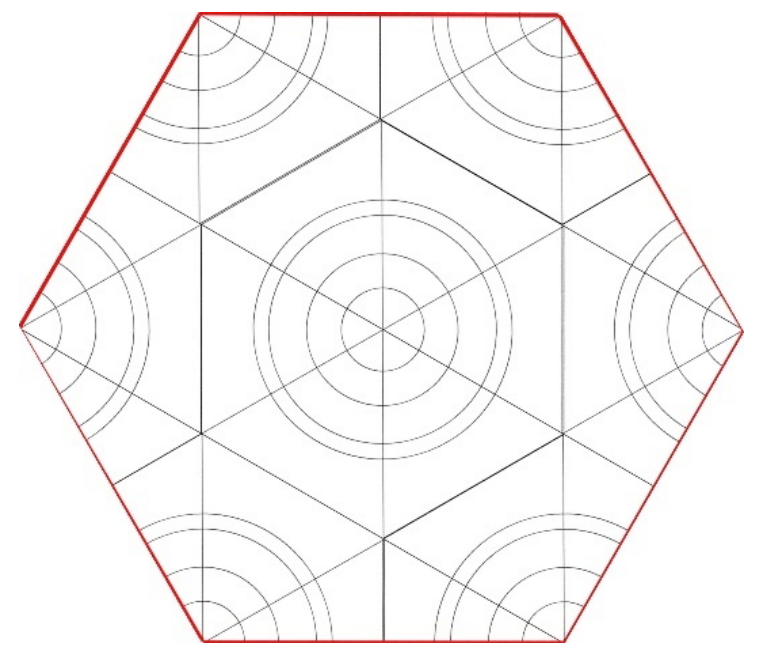

Figure 3-3. Super-Cell Problem of 2 Rings of Identical Hexagonal Compact-Cells. 
Table 3-3. MC ${ }^{2}-3$ Eigenvalue Results vs. Radial Meshes of Unit-Cell Problem

\begin{tabular}{|c|c|c|c|c|c|c|c|}
\hline \multirow{2}{*}{$\begin{array}{c}\text { Azimuthal } \\
\text { angles }^{\mathbf{a}}\end{array}$} & $\begin{array}{c}\text { Ray spacing } \\
\mathbf{( c m )}\end{array}$ & \multicolumn{5}{|c|}{ Number of radial meshes (fuel compact, graphite moderator) } \\
\cline { 3 - 8 } & $\mathbf{3 , 5}$ & $\mathbf{5 , 5}$ & $\mathbf{7 , 5}$ & $\mathbf{9 , 5}$ & $\mathbf{7 , 7}$ & $\mathbf{7 , 9}$ \\
\hline \multirow{2}{*}{30} & 0.05 & 1.24420 & 1.24424 & 1.24411 & 1.24421 & 1.24407 & 1.24406 \\
\cline { 2 - 8 } & 0.02 & - & 1.24314 & - & - & - & - \\
\hline
\end{tabular}

a Number of azimuthal angles in 180 degrees.

Table 3-4. MC²-3 Eigenvalue Results of Super-Cell Problem

\begin{tabular}{|c|c|c|c|c|c|c|c|}
\hline Azimuthal & Ray spacing & \multicolumn{5}{|c|}{ Number of radial meshes (fuel compact, graphite moderator) } \\
\cline { 2 - 8 } angles $^{\mathbf{a}}$ & $\mathbf{( c m )}$ & $\mathbf{3 , 5}$ & $\mathbf{5 , 5}$ & $\mathbf{7 , 5}$ & $\mathbf{9 , 5}$ & $\mathbf{7 , 7}$ & $\mathbf{7 , 9}$ \\
\hline 18 & 0.05 & 1.24397 & 1.24395 & 1.24377 & 1.24365 & 1.24375 & 1.24376 \\
\hline \multirow{3}{*}{30} & 0.05 & - & 1.24294 & - & - & - & - \\
\cline { 2 - 8 } & 0.02 & - & 1.24414 & - & - & - & - \\
\cline { 2 - 8 } & 0.01 & - & 1.24374 & - & - & - & - \\
\hline 66 & 0.02 & - & 1.24395 & - & - & - & - \\
\hline
\end{tabular}

a Number of azimuthal angles in 180 degrees.

In the super-cell problem of 5 radial meshes in each of fuel compact and moderator, the reduction of ray spacing from $0.02 \mathrm{~cm}$ to $0.01 \mathrm{~cm}$ (with 30 azimuthal angles) decreases the reactivity by 26 pcm. The increase in the number of azimuthal angles from 30 to 66 (with $0.02 \mathrm{~cm}$ ray spacing) decreases the reactivity by $12 \mathrm{pcm}$. The increase in the number of radial meshes in fuel compact from 5 to 7 (with 5 radial meshes for moderator) decreases the reactivity by $12 \mathrm{pcm}$, and the increase from 7 to 9 decreases the reactivity by $8 \mathrm{pcm}$. No noticeable difference is observed in reactivity when the number of meshes in the moderator region is increased from 5 to 9 . Based on these results, the $\mathrm{MC}^{2}-3$ calculations were performed with the super-cell model of 7 and 5 equal volume radial meshes in the fuel and moderator regions, respectively, and 66 azimuthal angles in 180 degrees and a ray spacing of $0.01 \mathrm{~cm}$. A convergence criterion of $10^{-6}$ was used for both eigenvalue and fission source.

\subsection{Comparison of Eigenvalue Results}

For the super-cell model shown in Figure 3-3, $\mathrm{MC}^{2}-3$ calculations were performed using the three methods: the SCALE method, the Sanchez-Pomraning method, and the ILSS method developed in this study. Since the Sanchez-Pomraning method can consider the anisotropic scattering only in the graphite matrix region [11] and a numerical test showed that the anisotropic scattering effect is weak for this problem set, the performances of three methods were compared using the transport corrected $\mathrm{P}_{0}$ scattering option only.

Figure 3-4 compares the eigenvalues of $\mathrm{MC}^{2}-3 \mathrm{HFG}$ calculations with the transport corrected $\mathrm{P}_{0}$ scattering with the Serpent-2 results. The eigenvalues and reactivity errors of $\mathrm{MC}^{2}-3$ UFG and HFG calculations with the transport corrected $\mathrm{P}_{0}$ scattering option are compared with the Serpent-2 results in Tables 3-5 and 3-6. The eigenvalue difference of about $100 \mathrm{pcm}$ is observed between the UFG and HFG calculation results for high packing fraction cases. The seemingly good results of the UFG calculation would be the consequence of error cancellation. For heterogeneity effect, no noticeable difference was observed between the UFG and HFG calculations. Among the three methods, the ILSS method predicts the heterogeneity effect slightly better than the other two 
existing methods. The SCALE and the Sanchez-Pomraning methods tend to underestimate the heterogeneity effect by 150 and $100 \mathrm{pcm}$, respectively, for middle and high packing fraction problems, while the ILSS method slightly overestimates the heterogeneity effect by $70 \mathrm{pcm}$. One of the reasons for these trends is that the regular array of particles overestimates collisions in particles than the random configuration of particles [12]. Note that the particle unit-cell model of the SCALE method is the regular array model and the reason for underestimated eigenvalues is due to more U-238 capture rates in particles. The good results from the ILSS method indicate that the stochastic configuration of particles can be well represented by the homogenized compact with local spatial self-shielding in each particle.

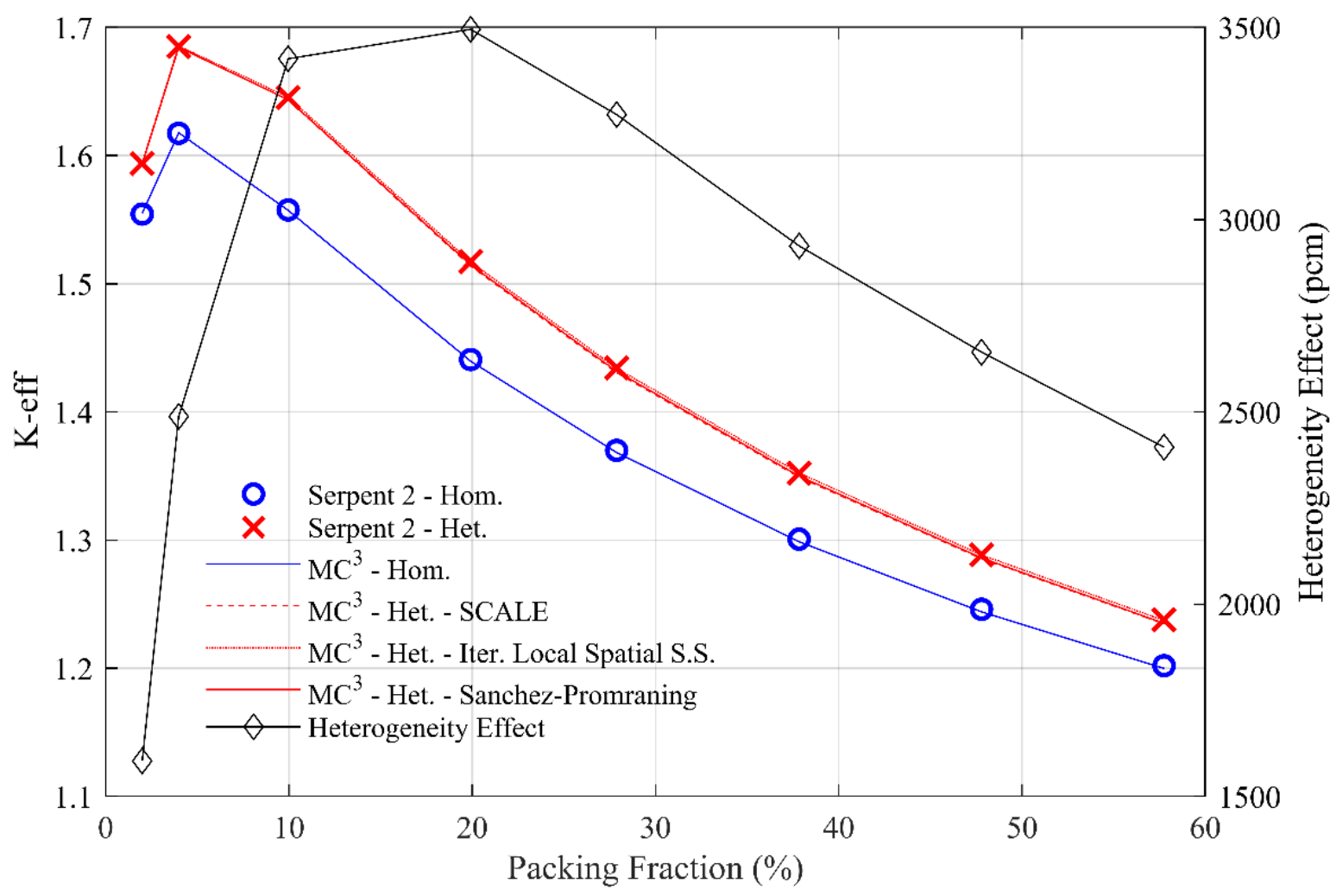

Figure 3-4. Comparison of Eigenvalues of $\mathrm{MC}^{2}-3$ HFG Calculation with Transport Corrected $\mathrm{P}_{0}$ Scattering with Serpent-2 Eigenvalue. 
Table 3-5. Results of MC $^{2}$-3 UFG Calculations with Transport Corrected $P_{0}$ Option for Fuel Compact Super-Cell Problems

\begin{tabular}{|c|c|c|c|c|c|c|c|c|c|c|c|c|c|c|}
\hline \multirow{4}{*}{$\begin{array}{c}\text { Packing } \\
\text { fraction } \\
(\%)\end{array}$} & \multirow{3}{*}{\multicolumn{2}{|c|}{$\begin{array}{l}\text { Homogenized } \\
\text { Compact Problem }\end{array}$}} & \multicolumn{12}{|c|}{ Heterogeneous Compact Problem } \\
\hline & & & \multicolumn{12}{|c|}{ Methods in $\mathrm{MC}^{2}-3$} \\
\hline & & & \multicolumn{4}{|c|}{ SCALE } & \multicolumn{4}{|c|}{ Iterative Local Spatial S.S. } & \multicolumn{4}{|c|}{ Sanchez-Pomraning } \\
\hline & K-eff & $\Delta \rho(\mathrm{pcm})$ & K-eff & $\begin{array}{c}\Delta \rho \\
(p c m)\end{array}$ & $\begin{array}{c}\text { Het. } \\
\text { Effect } \\
\text { (pcm) }\end{array}$ & $\begin{array}{l}\Delta \text { Het. } \\
\text { Effect } \\
(\text { pcm) }\end{array}$ & K-eff & $\begin{array}{c}\Delta \rho \\
(\mathrm{pcm})\end{array}$ & $\begin{array}{c}\text { Het. } \\
\text { Effect } \\
(p c m)\end{array}$ & $\begin{array}{l}\Delta \text { Het. } \\
\text { Effect } \\
\text { (pcm) }\end{array}$ & K-eff & $\begin{array}{c}\Delta \rho \\
(\mathrm{pcm})\end{array}$ & $\begin{array}{c}\text { Het. } \\
\text { Effect } \\
\text { (pcm) }\end{array}$ & $\begin{array}{l}\Delta \text { Het. } \\
\text { Effect } \\
(\text { pcm) }\end{array}$ \\
\hline 2.0 & 1.55445 & 8 & 1.59358 & -5 & 1580 & -13 & 1.59374 & 1 & 1586 & -7 & 1.59315 & -22 & 1563 & -30 \\
\hline 4.0 & 1.61753 & 8 & 1.68456 & -19 & 2460 & -27 & 1.68524 & 5 & 2484 & -3 & 1.68409 & -35 & 2443 & -43 \\
\hline 10.0 & 1.55765 & 5 & 1.64323 & -71 & 3344 & -76 & 1.64528 & 5 & 3419 & 0 & 1.64330 & -69 & 3346 & -74 \\
\hline 19.9 & 1.44067 & -11 & 1.51409 & -134 & 3366 & -123 & 1.51716 & 0 & 3500 & 10 & 1.51485 & -101 & 3399 & -90 \\
\hline 27.9 & 1.36974 & -10 & 1.43116 & -156 & 3133 & -146 & 1.43439 & 1 & 3291 & 11 & 1.43202 & -114 & 3175 & -104 \\
\hline 37.8 & 1.30042 & -18 & 1.34987 & -132 & 2817 & -115 & 1.35297 & 37 & 2987 & 55 & 1.35060 & -92 & 2857 & -74 \\
\hline 47.8 & 1.24589 & -14 & 1.28644 & -138 & 2530 & -124 & 1.28927 & 33 & 2701 & 47 & 1.28695 & -107 & 2561 & -93 \\
\hline 57.8 & 1.20185 & -1 & 1.23574 & -135 & 2282 & -134 & 1.23827 & 31 & 2447 & 31 & 1.23603 & -116 & 2301 & -115 \\
\hline
\end{tabular}

* All $\Delta$ quantities are differences compared to Serpent-2 results.

Table 3-6. Results of MC $^{2}-3$ HFG Calculations with Transport Corrected $P_{0}$ Option for Fuel Compact Super-Cell Problems

\begin{tabular}{|c|c|c|c|c|c|c|c|c|c|c|c|c|c|c|}
\hline \multirow{4}{*}{$\begin{array}{c}\text { Packing } \\
\text { fraction } \\
(\%)\end{array}$} & \multirow{3}{*}{\multicolumn{2}{|c|}{$\begin{array}{c}\text { Homogenized } \\
\text { Compact Problem }\end{array}$}} & \multicolumn{12}{|c|}{ Heterogeneous Compact Problem } \\
\hline & & & \multicolumn{12}{|c|}{ Methods in MC2-3 } \\
\hline & & & \multicolumn{4}{|c|}{ SCALE } & \multicolumn{4}{|c|}{ Iterative Local Spatial S.S. } & \multicolumn{4}{|c|}{ Sanchez-Pomraning } \\
\hline & K-eff & $\Delta \rho(p \mathrm{~cm})$ & K-eff & $\begin{array}{c}\Delta \rho \\
(\mathrm{pcm})\end{array}$ & $\begin{array}{c}\text { Het. } \\
\text { Effect } \\
\text { (pcm) }\end{array}$ & $\begin{array}{l}\Delta \text { Het. } \\
\text { Effect } \\
(p c m)\end{array}$ & K-eff & $\begin{array}{c}\Delta \rho \\
(\mathrm{pcm})\end{array}$ & $\begin{array}{c}\text { Het. } \\
\text { Effect } \\
\text { (pcm) }\end{array}$ & $\begin{array}{l}\Delta \text { Het. } \\
\text { Effect } \\
\text { (pcm) }\end{array}$ & K-eff & $\begin{array}{c}\Delta \rho \\
(\mathrm{pcm})\end{array}$ & $\begin{array}{c}\text { Het. } \\
\text { Effect } \\
(p c m)\end{array}$ & $\begin{array}{l}\Delta \text { Het. } \\
\text { Effect } \\
(\text { pcm) }\end{array}$ \\
\hline 2.0 & 1.55440 & 6 & 1.59355 & -7 & 1581 & -12 & 1.59379 & 3 & 1590 & -3 & 1.59331 & -16 & 1571 & -22 \\
\hline 4.0 & 1.61741 & 3 & 1.68448 & -22 & 2462 & -25 & 1.68523 & 5 & 2488 & 1 & 1.68433 & -27 & 2456 & -30 \\
\hline 10.0 & 1.55735 & -7 & 1.64293 & -82 & 3345 & -75 & 1.64504 & -4 & 3423 & 3 & 1.64351 & -61 & 3366 & -53 \\
\hline 19.9 & 1.44013 & -37 & 1.51349 & -160 & 3366 & -124 & 1.51663 & -23 & 3503 & 13 & 1.51471 & -107 & 3419 & -70 \\
\hline 27.9 & 1.36903 & -48 & 1.43040 & -193 & 3134 & -145 & 1.43372 & -32 & 3296 & 16 & 1.43163 & -133 & 3194 & -85 \\
\hline 37.8 & 1.29956 & -69 & 1.34898 & -181 & 2819 & -112 & 1.35219 & -5 & 2995 & 63 & 1.34996 & -127 & 2873 & -59 \\
\hline 47.8 & 1.24491 & -77 & 1.28545 & -198 & 2533 & -120 & 1.28841 & -19 & 2712 & 58 & 1.28613 & -157 & 2574 & -79 \\
\hline 57.8 & 1.20078 & -75 & 1.23468 & -204 & 2287 & -129 & 1.23735 & -29 & 2461 & 45 & 1.23509 & -177 & 2313 & -102 \\
\hline
\end{tabular}

* All $\Delta$ quantities are differences compared to Serpent-2 results. 


\section{Conclusions and Future Work}

The existing self-shielding methods of treating the double heterogeneity for TRISO fuel cross section generation (the SCALE method and the Sanchez-Pomraning method) have been studied and implemented in $\mathrm{MC}^{2}-3$ to assess their performances. In addition, a new method named the iterative local spatial self-shielding (ILSS) method was developed and implemented in $\mathrm{MC}^{2}-3$ to obtain more accurate self-shielded cross sections for TRISO fuel.

The new method approximately takes into account the effect of randomly distributed particles on the particle shadowing effect using a homogenized compact region surrounding a particle of interest at the center. The self-shielded cross sections of the particle at the center are determined iteratively since the cross sections of the homogenized compact region are calculated using them. For the energy range above $100 \mathrm{keV}$ where the fuel-to-moderator ratio is more important than the random distribution of particles, a single particle unit-cell model is used by preserving the average amount of moderator per fuel particle in the system.

Verification tests were performed with 2D super-cell compact problems for prismatic type of very high temperature reactor (VHTR) at different packing fractions from $2.0 \%$ to $57.8 \%$. The $\mathrm{MC}^{2}-3$ results were compared with the continuous energy Monte Carlo solutions obtained with the sample average of 25 independent runs of Serpent- 2 using different random configurations of particles. UFG and HFG calculations were performed with the transport corrected $\mathrm{P}_{0}$ scattering option. Both UFG and HFG calculations showed almost the same heterogeneity effects. Among the three methods, the new method produced slightly better results than the other two methods. The SCALE and the Sanchez-Pomraning methods tend to underestimate the heterogeneity effect by 150 and 100 $\mathrm{pcm}$, respectively, while the new method overestimates the heterogeneity effect by $70 \mathrm{pcm}$.

As future work, the new self-shielding method will be extended to perform pebble calculations and compare results with those from the SCALE and Sanchez-Pomraning methods. Furthermore, the new method will be optimized to be made practically applicable to on-the-fly resonance treatment for lattice or whole-core calculations for advanced reactor applications. 


\section{REFERENCES}

1. C.H. Lee and Y.S. Jung, “Generation of the Cross Section Library for PROTEUS,” ANL/NE18-2, Argonne National Laboratory, January (2018).

2. C.H. Lee and W.S. Yang, "MC²-3: Multigroup Cross Section Generation Code for Fast Rector Analysis,” Nucl. Sci. Eng., 187, 268 (2017).

3. Y.S. Jung et al., "PROTEUS-MOC User Manual," ANL/NSE-18/10, Argonne National Laboratory (2018).

4. C.H. Lee et al., Argonne National Laboratory, unpublished information, July (2020).

5. M.L. Williams, "Resonance Self-shielding Methodologies in SCALE 6,” Nucl. Technol., 174, 149-168 (2011).

6. R. Sanchez, G.C. Pomraning, "A Statistical Analysis of the Double Heterogeneity Problem,” Ann. Nucl. Energy, 18, 371-395 (1991).

7. R. Sanchez, "Renormalized Treatment of the Double Heterogeneity with the Method of Characteristics,” Proc. PHYSOR 2004, Chicago, Illinois, April 25-29 (2004).

8. M.L. Williams and M. Asgari, "Computation of Continuous-Energy Neutron Spectra with Discrete Ordinates Transport Theory,” Nucl. Sci. Eng., 121, 173-201 (1995).

9. J. Leppänen et al., "The Serpent Monte Carlo Code: Status, Development, and Applications in 2013,” Ann. Nucl. Energy, 82, 142-150 (2015).

10. R.C. Potter et al., "NGNP Preliminary Point Design - Results of the Initial Neutronics and Thermal-Hydraulic Assessments,” INEEL/EXT-03-00870 Rev. 1, Idaho National Laboratory, September (2003).

11. R. Sanchez, Personal Communication, June (2020).

12. T.K. Kim et al., "Preliminary Assessment of Lattice Physics Capabilities for VHTR Analysis," Transactions of ANS Winter Meeting and Nuclear Technology Expo, Washington, D.C., June 21, pp. 543-544 (2004). 
Argonne

Nuclear Science and Engineering Division

Argonne National Laboratory

9700 South Cass Avenue, Bldg. 208

Argonne, IL 60439-4842

www.anl.gov 